\title{
EBAP Regimen
}

National Cancer Institute

\section{Source}

National Cancer Institute. EBAP Regimen. NCI Thesaurus. Code C9586.

A chemotherapy regimen consisting of vindesine, carmustine, doxorubicin, and prednisone that may be used in the treatment of recurrent lymphomas. 